\title{
A case of cerebellar cognitive affective syndrome following left paramedian cerebellar tumor
}

Oluwole Jegede1, Eshban Emmanuel2, Oyelakin Ayotomide1, Jaspreet Gill3, Jisha Kallikkadan1, Gurneet Matharoo4, Anna Volkovinskaia3, Thayaparan Balakumar4, Olawale 0jo1, Shantale Williams ${ }^{4}$, Anam Shaikh'5, Sherina Langdon ${ }^{4}$, Heela Azizi ${ }^{4}$, Alexa Kahn', Dina Rimawi ${ }^{4}$, Tasmia Khan², Mandana Ashrafi', Mutahher Muhammed 4 , Kodjovi Kodjo', Ivan Leung 3 , Carolina Nisenoff' 1 and Ayodeji Jolayemi1

* Correspondence

ojegede@interfaithmedical.com

Oluwole Jegede

1Department of Psychiatry and Behavioral Sciences, Interfaith Medical Center, Brooklyn, New York

2Medical University of the Americas

3Saba University School of Medicine ${ }^{4}$ American University of Antigua College of Medicine

${ }^{5}$ St. Matthews University School of Medicine

Received: May 28, 2018

Accepted: Jun 15, 2018

Published: Jun 21, 2018

\begin{abstract}
We report the case of a 49-year-old man who presented to our psychiatric emergency room with acute agitation and disorganized behavior. The patient's symptoms were an exacerbation of his reported chronic mood disorder concurrent with left paramedian cerebellar mass for which he underwent a surgical resection several years earlier. Associated with his acute psychiatric symptoms were findings of impaired cognition and altered perception. Diagnostic MRI done during index acute presentation revealed evidence of cerebellar mass regrowth. The disorders of cognition, mood and perceptions which involved a concurrent insult to the cerebellum is consistent with the diagnosis of Cerebellar Cognitive Affective Syndrome (CCAS). Our case underscores and strengthens the notion that the cerebellum plays a significant role in cognitive, mood and perceptual disturbances.
\end{abstract}

Keywords: Cerebellar cognitive affective syndrome, Cerebellum, depression, Cognitive dysfunction, Affective dysregulation.

\section{Introduction}

The function of the cerebellum is commonly understood as one of motor coordination. However, motor discoordination is not the only known manifestation of cerebellar injury. Cerebellar Cognitive Affective Syndrome (CCAS) describes cognitive impairments, mood disorder, and perceptual disturbances in the setting of cerebellar insult [1]. Tavano et al compared the various psychiatric features in 27 patients with Cerebellar insult. The patients in the study by Tavano et al showed similar affective symptoms including withdrawal, behavioral stereotypies, aggressiveness, and self-injury. Schmahmann identified four clinical features of CCAS namely: personality changes, impairments in executive functioning, visual-spatial cognitions and impairments in language [2].
Recent neuroimaging findings and case series have pointed to possible cognitive and affective roles of the cerebellum. The emergence of new brain imaging technology began in the early 1980s and transformed research conducted in measuring brain activity. Tools such as Positron Emission Tomography (PET) and functional Magnetic Resonance Imaging (fMRI) have been used to observe cerebellar function as cognitive stimuli were elicited. Peterson and his colleagues demonstrated that different parts of the cerebellum are responsible for motor and cognitive functions [3]. They observed cerebellar activity via PET scan while subjects performed cognitive and motor tasks. These observations revealed increased activity in the anterior lobe of the cerebellum during motor tasks and increased activity in the lateral lobe of the cerebellum during cognitive tasks [3]. As a 
result, the classic view of cerebellar function is being increasingly redefined over the past few decades.

We report a case of a 49-year-old man with a tumor in the left paramedian cerebellum presenting with significant mood symptoms consistent with CCAS and supporting the likely role of the cerebellum in cognition, mood and perception.

\section{Case Presentation}

The patient is a 49-year-old Hispanic man with a history Substance Use Disorder in full remission for 14 years and a partial resection of a paramedian cerebellar tumor. He was brought to the hospital emergency department due to aggression and agitation in the context of alcohol intoxication. He reports a chronically depressed mood over the past four years, with worsening symptoms over the past four weeks before presentation. His worsening of mood symptoms includes ultra-rapid mood swings within the same day from depressed to euthymic to irritable. He reported worsening chronic auditory and visual hallucinations over the same four-week period. Other associated symptoms of mood disturbance include hopelessness, poor self-worth, low energy, anhedonia, poor concentration, and insomnia. These symptoms occurred in the absence of alcohol intoxication, which he stated he began using compulsively two weeks prior to index presentation.

He was diagnosed with dysthymia at the age of 44 years and recalled associated blurring of vision and new-onset intention tremor around the same time. His symptoms worsened, with onset of lack of concentration, loss of short-term memory, severe headaches, rapid mood swings, visual and auditory hallucinations. A year later, an MRI showed a $1.7 \mathrm{~cm}$ $x 8.6 \mathrm{~cm}$ paramedian cerebellar mass for which he had a partial resection. He was able to resume his normal lifestyle with some resolution of his mood symptoms.

Post-surgical resection until current presentation, he developed increasing dizziness and difficulties with balance and memory. Furthermore, his depression had worsened with recurrence of perceptual disturbances of a visual nature. His presentation was diagnosed as a case of Schizoaffective disorder and he was managed on
Risperidone, Trazodone, Divalproex sodium and Mirtazapine.

On mental status examination, the patient's affect was flat and depressed with slow speech, he also had significant psychomotor retardation. His thought process was linear and goal-directed. He scored 17/30 on the Montreal Cognitive Assessment (MOCA). He had notable deficits in the visuospatial and executive function, attention (in particular serial 7 subtraction), language, delayed recall, and affect. Neurological examination revealed an intact cranial nerves (CN I - CN XII) with a positive Romberg's test. Patient swayed to the right on tandem gait test and showed no evidence of dysmetria on finger-to-nose testing. In addition, he showed no signs of dysdiadochokinesia. There was no pronator drift of outstretched arms. Rapid alternating movements and fine finger movements were performed with difficulty. Hematology and chemistry were within normal limits with a blood alcohol level of $250 \mathrm{mg} / \mathrm{dl}$ on admission. MRI on presentation revealed an acute recurrence of his tumor as shown in Figures 1.

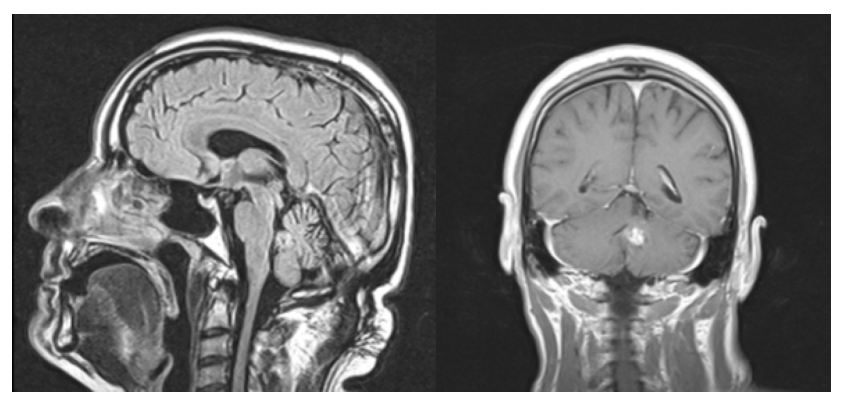

Figure 1. showing MRI with contrast showing $1.5 \mathrm{~cm}$ enhancing lesion in left medial cerebellum consistent with residual or recurrent mass.

\section{Discussion}

The cerebellum consists of many cerebro-cerebellar connections with the frontal, parietal, temporal, and occipital lobes. Efferents from the cerebellar nuclei project to multiple areas of the thalamus and from the thalamus to neocortical areas: premotor, prefrontal, and posterior parietal areas of the cerebral cortex. Observations by Snider, suggest that the cerebellum also projects fibers to the locus ceruleus and ventral tegmental area, signifying that cerebellar projections have the ability to reach the limbic system [4]. Other studies have shown projections of the Papez Circuit to ventral tegmental 
area, interpeduncular area, and periaqueductal gray areas. These areas also receive projections from the cerebellar nuclei.

A review of studies investigating cerebellar volumes in patients with bipolar disorder and major depressive disorder (MDD) compared with healthy controls showed significant reduction of cerebellar volumes in the latter (particularly in the vermal sub-region), the authors also found that the severity of bipolar symptoms was associated with increased vermal damage [5]. Among patients with schizophrenia, a diminished blood flow was noticed in the cerebellar cortex and vermis during performance of cognitive tasks such as attention and memory [6].

Schmahmann et al observed deficits seen in the majority of patients he studied, deficit areas included executive function, visuospatial integration, naming, mathematical skills, behavior and personality adjustment [7]. Our case showed similar deficits as well pairticularly in the visuospatial and executive function, attention, language, delayed recall, and affect domains of the MSE and MOCA. The patient also demonstrated significant mood and perceptual disturbances of a visual nature concurrent with findings of left paramedian cerebellar tumor. This is consistent with Tavano, who identified cognitive impairments, mood disorder, and perceptual disturbances in the setting of cerebellar insult [1]. Of particular clinical significance in our patient is the partial resolution of symptoms after his initial surgery and the recurrence of similar affective and psychotic symptoms with recurrence of his cerebellar tumor. We found it as clinically uncanny not only that our patient has a recurrent cerebellar tumor but that the tumor is located in the left paramedian region.

\section{Conclusion}

The association of cerebellar dysfunction to cognitive, affective, and perceptual disturbances, although not a new concept is making its way back in relatively new literature [8]. The present case provides further support for the association of cerebellar dysfunction with disturbances in affect, perception and cognition [9-18].

\section{Author disclosures}

The authors declare that they have no competing interests.

\section{Copyrights}

( 2018 Oluwole Jegede et al.; licensee OA Journal of Neuropsychiatry. This is an Open Access article distributed under the terms of the Creative Commons Attribution License (http:// creativecommons.org/licenses/by/4.0).

\section{References}

1. Tavano A, Grasso R, Gagliardi C, et al. (2007) Disorders of cognitive and affective development in cerebellar malformations. Brain. 130: 2646-2660.

2. Schmahmann JD, Sherman JC (1998) The cerebellar cognitive affective syndrome. Brain. 121: 561-579.

3. Petersen SE, Fox PT, Posner MI, et al. (1989) Positron emission tomographic studies of the processing of single words. J Cogn Neurosci. 1: 153-170.

4. Snider RS, Maiti A (1976) Cerebellar contributions to the papez circuit. J Neurosci Res. 2: 133-146.

5. Soares JC, Mann JJ (1997) The anatomy of mood disorders review of structural neuroimaging studies. BiolPsychiatry. 41: 86-106.

6. Crespo-Facorro B, Barbadillo L, Pelayo-Teran JM, et al. (2007) Neuropsychological functioning and brain structure in schizophrenia. Int Rev Psychiatry. 19:325-336

7. Schmahmann JD (2004) Disorders of the cerebellum: ataxia, dysmetria of thought, and the cerebellar cognitive affective syndrome. J Neuropsychiatry Clin Neurosci. 16: 367-378.

8. Bostan AC, Dum RP, Strick PL (2013) Cerebellar networks with the cerebral cortex and basal ganglia. Trends in Cognitive Sciences. 17: 241-254.

9. De Smet HJ, Paquier P, Verhoeven J, et al. (2013) The cerebellum: its role in language and related cognitive and affective functions. Brain \& Language. 127: 334-342.

10. Wolf U, Rapoport M, Tom Schweizer F (2009) Evaluating the Affective Component of the Cerebellar Cognitive Affective Syndrome. J Neuropsychiatry and Clinical Sciences. 21: 245-253.

11.Graf H, Franke B, Abler B (2013) Cerebellar Cognitive Affective Syndrome in Dandy-Walker Variant Disorder. J Neuropsychiatry Clin Neurosci 25: 3.

12.Schmahmann JD (2010) The role of the cerebellum in cognition and emotion: personal reflections since 1982 on the dysmetria of thought hypothesis, and its historical evolution from theory to therapy. Neuropsychol Rev. 20: 236-260.

13.Phillips JR, Hewedi DH, Eissa AM, et al. (2015) The cerebellum and psychiatric disorders. Front Public Health. 3: 66.

14.Baldaçara L, Borgio JGF, de Lacerda ALT, et al. (2008) Cerebellum and psychiatric disorders. Rev Bras Psiquiatr. 30: 281-289.

15.Konarski JZ, McIntyre RS, Grupp LA, et al. (2005) Is the cerebellum relevant in the circuitry of neuropsychiatric disorders? J Psychiatry Neurosci. 30: 178-186.

16.Omar D, Ryan T, Carson A, et al. (2014) Clinical and methodological confounders in assessing the cerebellar cognitive affective syndrome in adult patients with posterior fossa tumours. Br J Neurosurg. 28: 755-764.

17.Brossard-Racine M, du Plessis AJ, Limperopoulos C (2015) Developmental cerebellar cognitive affective syndrome in expreterm survivors following cerebellar injury. Cerebellum. 14 151-164.

18. McCaffrey P, McColl D, Blackmon R (2001) Basic and Clinical Neuroscience of Communication Disorders. American Speech Language Hearing Association Conference, New Orleans. 